Pacific Journal of Mathematic 


\title{
ON NORMED RINGS WITH MONOTONE MULTIPLICATION
}

\section{Silvio Aurora}

\begin{abstract}
It is shown that if a normed division ring has a norm which is "multiplication monotone" in the sense that $N(x)<$ $N\left(x^{\prime}\right)$ and $N(y)<N\left(y^{\prime}\right)$ imply $N(x y) \leqq N\left(x^{\prime} y^{\prime}\right)$, and if the norm is "commutative" in the sense that $N(\cdots x y \cdots)=N(\cdots y x \cdots)$ for all $x$ and $y$, then the topology of that ring is given by an absolute value. A consequence of this result is that if the norm of a connected normed ring with unity is multiplication monotone and commutative then the ring is embeddable in the system of quaternions.
\end{abstract}

Pontrjagin has shown [7] that the only locally compact connected fields are the field of real numbers and the field of complex numbers. A theorem of A. Ostrowski [6] implies that if the topology of a connected field is given by an absolute value then the field is (isomorphic to) a subfield of the field of complex numbers. Both results are contributions toward the solution of the problem of determining what connected fields exist.

In this note the more restricted question of studying connected normed fields is considered. (It is recalled that a normed ring has its topology induced by a norm function $N$; that is, $N$ is a real-valued function defined on the ring such that: (i) $N(0)=0$ and $N(x)>0$ for $x \neq 0$, (ii) $N(-x)=N(x)$ for all $x$, (iii) $N(x+y) \leqq N(x)+N(y)$ for all $x$ and $y$, (iv) $N(x y) \leqq N(x) N(y)$ for all $x$ and $y$.) Ostrowski's results may be regarded as the treatment of the special case of this problem in which the norm $N$ satisfies the additional condition

$$
N(x y)=N(x) N(y)
$$

for all $x$ and $y$. This extra requirement is replaced here by the weaker condition that $N$ be multiplication monotone in the sense that whenever $N(x)<N\left(x^{\prime}\right)$ and $N(y)<N\left(y^{\prime}\right)$ then $N(x y) \leqq N\left(x^{\prime} y^{\prime}\right)$.

Specifically, it is shown in the corollary of Theorem 3 that if a commutative connected normed ring with unity has a multiplication monotone norm then that ring is (algebraically and topologically isomorphic to) a subring of the field of complex numbers. (The version of this statement which appears below actually includes the noncommutative case as well.) The basic device employed in obtaining this result is Theorem 2, which asserts that if a normed division ring has a multiplication monotone norm $N$ such that

$$
N(\cdots x y \cdots)=N(\cdots y x \cdots)
$$


for all $x$ and $y$ then there is an absolute value which induces the topology of the ring.

2. Preliminaries. It is recalled that a norm for a ring $A$ is a real-valued function $N$ on $A$ such that: (i) $N(0)=0$ and $N(x)>0$ for all nonzero $x$ in $A$, (ii) $N(-x)=N(x)$ for all $x$ in $A$, (iii) $N(x+y) \leqq$ $N(x)+N(y)$ for all $x, y$ in $A$, (iv) $N(x y) \leqq N(x) N(y)$ for all $x, y$ in $A$. If a norm $N$ for a ring $A$ also has the property that $N(x y)=N(x) N(y)$ for all $x, y$ in $A$ then $N$ is called an absolute value for $A$.

By a normed ring is meant a ring $A$, together with a norm $N$ for $A$. The norm for a normed ring induces a metric, and therefore a topology, in $A$.

A topological ring is called a $Q$-ring of its set of quasiinvertible elements is open; for a topological ring $A$ with unity to be a $Q$-ring it is necessary and sufficient that the set of invertible elements be open. In particular, it can be shown that every complete normed ring with unity is a Q-ring.

Further details on these concepts can be found in [1] and [4], where the term metric ring is employed for a normed ring.

If a norm $N$ for a ring $A$ has the property that $N(\cdots x y \cdots)=$ $N(\cdots y x \cdots)$ for all $x, y$ in $A$ then $N$ will be called a commutative norm. For instance, absolute values are always commutative, and every norm for a commutative ring is also commutative.

In addition to the above notions, we shall also refer to the concepts which figure in [5], and we shall make use of the criteria given by Kaplansky in that paper for a topological division ring to admit an equivalent absolute value.

Two elementary lemmas will help to translate Kaplansky's criteria to the special case of normed division rings. The proofs are routine.

Lemma 1. An element $x$ of a normed ring is topologically nilpotent if and only if there exists a natural number $n$ such that $N\left(x^{n}\right)<1$.

Lemma 2. The set of topologically nilpotent elements of a normed ring is open.

Kaplansky's criteria can now be rephrased to fit the needs of the present discussion.

THEOREM 1. Let $K$ be a normed division ring whose norm is commutative. In order for $K$ to admit an equivalent absolute value 
(that is, an absolute value whose induced topology coincides with the topology induced by the norm for $K$ ), it is necessary and sufficient that the set of elements which are either topologically nilpotent or neutral be right bounded.

Proof. The necessity of the conditions is obvious. For the sufficiency of the conditions, we first note that the commutativity of the norm implies that $N(x)=N(1)$ whenever $x$ is an element of the commutator subgroup of the multiplicative group of nonzero elements of $K$; this commutator subgroup is therefore metrically bounded and is consequently right bounded. Lemma 2 and [5; Th. 2] imply that there is an equivalent absolute value for $K$.

3. Rings with multiplication monotone norm. We shall subject the norm for a normed ring to a monotonicity condition which is of interest because it implies the existence of an absolute value equivalent to the given norm.

Definition. A norm $N$ for a ring $A$ is said to be multiplication monotone provided that whenever $N(x)<N\left(x^{\prime}\right)$ and $N(y)<N\left(y^{\prime}\right)$ then $N(x y) \leqq N\left(x^{\prime} y^{\prime}\right)$.

Clearly every absolute value is multiplication monotone, while the following theorem indicates that under suitable conditions a multiplication monotone norm for a division ring must have an equivalent absolute value.

THEOREM 2. Let $K$ be a normed division ring whose norm is commutative and multiplication monotone. Then there is an equivalent absolute value for $K$.

Proof. The theorem obviously holds for discrete division rings, so we may confine our attention to nondiscrete division rings.

Let $x$ be a fixed element of $K$ such that $0<N(x)<N(1)^{-1}$. Then if $N(y)>N\left(x^{-2}\right)$ it follows that $N\left(y^{-1}\right) \leqq N(x)<1$, and $y$ is therefore inversely nilpotent. Thus whenever $y$ is topologically nilpotent or neutral we have $N(y) \leqq N\left(x^{-2}\right)$, so that the set of elements of $K$ which are topologically nilpotent or neutral is metrically bounded and therefore right bounded. Theorem 1 yields the desired result.

It is possible to relax the requirement that the ring in question be a division ring, provided that the ring is connected. In order to achieve this we introduce the notion of generalized zero-divisors. 
Definition. An element $b$ of a normed ring $A$ will be called a generalized left zero-divisor (generalized right zero-divisor) provided that the greatest lower bound of the set $\{N(b x) / N(x) \mid x \neq 0\}(\{N(x b) /$ $N(x) \mid x \neq 0\}$ ) is zero.

These are essentially the definitions which were employed in [1], but we may also note that $b$ is a generalized left zero-divisor (generalized right zero-divisor) if and only if there exists a sequence $\left\{x_{n}\right\}$ of nonzero elements of $A$ such that

$$
\lim N\left(b x_{n}\right) / N\left(x_{n}\right)=0\left(\lim N\left(x_{n} b\right) / N\left(x_{n}\right)=0\right) .
$$

Although normed rings usually have many generalized zero-divisors it can be shown that a connected normed ring whose norm is multiplication monotone has no generalized zero-divisors other than zero.

Lemma 3. Let $A$ be a connected normed ring with unity such that the norm for $A$ is multiplication monotone. Then $A$ has no generalized left zero-divisors or generalized right zero-divisors other than zero.

Proof. Suppose $b$ is a generalized left zero-divisor in $A$. Let $\left\{x_{n}\right\}$ be a sequence of nonzero elements of $A$ such that

$$
\lim N\left(b x_{n}\right) / N\left(x_{n}\right)=0 .
$$

Choose a sequence $\left\{y_{n}\right\}$ in $A$ such that $(1 / 2) N\left(x_{n}\right)<N\left(y_{n}\right)<N\left(x_{n}\right)$ for every natural number $n$.

If $I$ is the set of all elements $c$ of $A$ such that

$$
\lim N\left(c y_{n}\right) / N\left(y_{n}\right)=0
$$

then $I$ is clearly a left ideal in $A$. Also, whenever $c$ is an element of $A$ such that $N(c)<N(b)$ then $N\left(c y_{n}\right) / N\left(y_{n}\right) \leqq N\left(b x_{n}\right) /\left((1 / 2) N\left(x_{n}\right)\right)$ for all $n$, so that $c$ is an element of $I$. Thus, if $b$ were not zero then an entire neighborhood of zero would be contained in the left ideal $I$, and $I$ would therefore be open and closed in the connected ring $A$; consequently $I$ would coincide with $A$, in contradiction to the fact that $I$ can not contain the unity of $A$. We conclude that $b$ is zero.

Similarly, every generalized right zero-divisor is zero.

In order to obtain the desired results concerning connected normed rings we first dispose of a special case in the following lemma.

Lemma 4. Let $A$ be a connected ring with unity such that the set $A^{\sharp}$ of nonzero elements of $A$ is disconnected. Then $A$ is a division ring. 
Proof. If $c$ is a nonzero element of $A$ then the mapping $x \rightarrow c x$ is clearly a continuous endomorphism of the additive group of $A$, so that its image $H$ is a connected nonzero subgroup of the additive group of $A$. But it can be shown that the additive group of $A$ is continuously isomorphic to the additive group of real numbers (for instance, a proof is outlined in [3; Chap. 5, p. 28, Exercise 4]), and $H$ must therefore coincide with the additive group of $A$. Thus, 1 is in $H$, so that $1=c d$ for some $d$ in $A$, and $c$ has a right inverse in $A$.

Since every nonzero element of $A$ has a right inverse in $A$ we conclude that $A$ is a division ring.

It is now possible to pass to the general case.

THEOREM 3. Let $K$ be a connected normed Q-ring with unity such that the norm for $K$ is commutative and multiplication monotone. Then $A$ is algebraically and topologically isomorphic to the field $\Re$ of real numbers, a dense connected subfield of the field $\mathfrak{c}$ of complex numbers, or a dense connected division subring of the division ring $\mathfrak{Q}$ of all real quaternions.

Proof. If the set $A^{\sharp}$ of nonzero elements of $A$ is not connected then Lemma 4 implies that $A$ is a division ring. On the other hand, if $A^{\ddagger}$ is connected then $A$ is a division ring according to [1; Th. 1] since Lemma 3 implies that $A$ has no generalized zero-divisors other than zero. In either case $A$ is a division ring.

There is an equivalent absolute value for the normed division ring $A$ by Theorem 2. Ostrowski's characterization of connected division rings with absolute value (see for instance [2; Th. 2, p. 131]) may then be applied to obtain the desired result.

COROLlary. Let $A$ be a connected normed ring with unity such that the norm for $A$ is commutative and multiplication monotone. Then $A$ is algebraically and topologically isomorphic to $\mathfrak{R}$, to a dense connected subring of $\mathfrak{C}$, or to a dense connected subring of $\Omega$.

The corollary is obtained by applying the theorem to the completion of $A$.

REMARK. Another kind of monotonicity condition could be introduced in normed division rings. The norm of a normed division ring can be described as inversion monotone provided that whenever $N(x)<N(y)$ for nonzero elements $x, y$ then $N\left(x^{-1}\right) \geqq N\left(y^{-1}\right)$. Theorem 2 remains valid if "multiplication monotone" is replaced by "inversion monotone" in the hypothesis, although some details of the proof must 
be modified. Similarly, the corollary of Theorem 3 continues to hold if "multiplication monotone" is replaced by "inversion monotone" in the statement of the corollary, provided that it is assumed that the ring is a division ring.

This note evolved from the consideration of some peripheral questions related to a problem which was investigated with the support of the Research Council of Rutgers University; the author wishes to express his appreciation to the Research Council for that support.

\section{REFERENCES}

1. S. Aurora, Multiplicative norms for metric rings, Pacific J. Math. 7 (1957), 12791304 .

2. N. Bourbaki, "Algèbre commutative," Chap. 5-6, Éléments de mathématique, Hermann, Paris, 1964.

3. ㄴ. "Topologie générale," Chap. 5-8, 3rd ed., Éléments de mathématique, Hermann, Paris, 1963.

4. I. Kaplansky, Topological rings, Amer. J. Math. 69 (1947), 153-183.

$5 . \quad$ - Topological methods in valuation theory, Duke Math. J. 14 (1947), 527541.

6. A. Ostrowski, Ueber einige Loesungen der Funktionalgleichung $\phi(x) \cdot \phi(y)=\phi(x y)$, Acta Math. 41 (1918), 271-284.

7. L. Pontrjagin, Ueber Stetige Algebraische Koerper, Ann. of Math. 33 (1932), 163174.

Received April 9, 1969.

RUTGERS UNIVERSITY

Newark, New Jersey 


\section{PACIFIC JOURNAL OF MATHEMATICS}

\section{EDITORS}

H. SAMELSON

Stanford University

Stanford, California 94305

\section{RichaRd PIERCe}

University of Washington

Seattle, Washington 98105
J. DUGUNDJI

Department of Mathematics

University of Southern California

Los Angeles, California 90007

RICHARD ARENS

University of California

Los Angeles, California 90024

\section{ASSOCIATE EDITORS}

E. F. BECKENBACH

B. H. NeUManN

F. WOLF

K. YosHIDA

\section{SUPPORTING INSTITUTIONS}

UNIVERSITY OF BRITISH COLUMBIA CALIFORNIA INSTITUTE OF TECHNOLOGY UNIVERSITY OF CALIFORNIA MONTANA STATE UNIVERSITY

UNIVERSITY OF NEVADA

NEW MEXICO STATE UNIVERSITY

OREGON STATE UNIVERSITY

UNIVERSITY OF OREGON

OSAKA UNIVERSITY

UNIVERSITY OF SOUTHERN CALIFORNIA
STANFORD UNIVERSITY

UNIVERSITY OF TOKYO

UNIVERSITY OF UTAH

WASHINGTON STATE UNIVERSITY

UNIVERSITY OF WASHINGTON

${ }^{*} \quad{ }^{*} \quad{ }^{*}$
AMERICAN MATHEMATICAL SOCIETY
CHEVRON RESEARCH CORPORATION
TRW SYSTEMS
NAVAL WEAPONS CENTER




\section{Pacific Journal of Mathematics}

\section{Vol. 33, No. $1 \quad$ March, 1970}

Mir Maswood Ali, On some extremal simplexes ................... 1

Silvio Aurora, On normed rings with monotone multiplication........... 15

Silvio Aurora, Normed fields which extend normed rings of integers....... 21

John Kelly Beem, Indefinite Minkowski spaces..................... 29

T. F. Bridgland, Trajectory integrals of set valued functions ........... 43

Robert Jay Buck, A generalized Hausdorff dimension for functions and sets ......................................... 69

Vlastimil B. Dlab, A characterization of perfect rings . . . . . . . . . . . . 79

Edward Richard Fadell, Some examples in fixed point theory ............ 89

Michael Benton Freeman, Tangential Cauchy-Riemann equations and uniform approximation ............................. 101

Barry J. Gardner, Torsion classes and pure subgroups ................ 109

Vinod B. Goyal, Bounds for the solution of a certain class of nonlinear

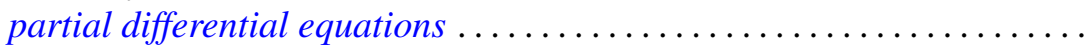

Fu Cheng Hsiang, On C, 1 summability factors of Fourier series at a given

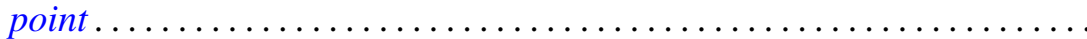

Lawrence Stanislaus Husch, Jr., Homotopy groups of PL-embedding

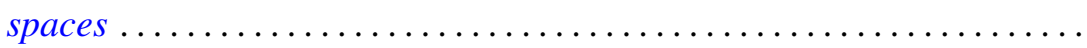

Daniel Ralph Lewis, Integration with respect to vector measures..........

Marion-Josephine Lim, $\mathscr{L}-2$ subspaces of Grassmann product spaces

Stephen J. Pierce, Orthogonal groups of positive definite multilinear functionals

W. J. Pugh and S. M. Shah, On the growth of entire functions of bounded index.

Siddani Bhaskara Rao and Ayyagari Ramachandra Rao, Existence of triconnected graphs with prescribed degrees . . .

Ralph Tyrrell Rockafellar, On the maximal monotonicity of subdifferential

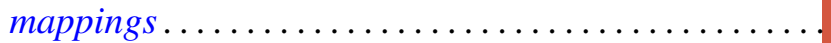

R. Shantaram, Convergence of a sequence of transformations of distribution functions. II ...............................

Julianne Souchek, Rings of analytic functions..............

Ted Joe Suffridge, The principle of subordination applied to functions of several variables...

Wei-lung Ting, On secondary characteristic classes in cobordism

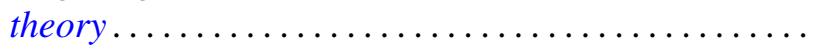

Pak-Ken Wong, Continuous complementors on $B^{*}$-algebras ...

Miyuki Yamada, On a regular semigroup in which the idempotents form a band. 